\title{
The chromatin remodeler LSH controls genome-wide cytosine hydroxymethylation
}

Maud de Dieuleveult ${ }^{1,4,}{ }^{\star}$, Martin Bizet ${ }^{1}$, Laurence Colin ${ }^{1}$, Emilie Calonne ${ }^{1}$, Martin Bachman $^{2}$, Chao Li ${ }^{3}$, Irina Stancheva ${ }^{3}$, François Fuks ${ }^{1^{*}}$ and Rachel Deplus ${ }^{1^{*}}$

${ }^{1}$ Laboratory of Cancer Epigenetics, Faculty of Medicine, ULB-Cancer Research Centre (U-CRC), Université Libre de Bruxelles, 808 route de Lennik, 1070 Brussels, Belgium.

${ }^{2}$ Medicines Discovery Catapult, Alderley Park, SK10 4 TG, UK

${ }^{3}$ Institute of Cell Biology, University of Edinburgh, Max Born Crescent, Edinburgh EH9 3BF

${ }^{4}$ Present address: Université de Paris, Institut Cochin, INSERM, U1016, CNRS, UMR8104, F-75014 PARIS, France

* To whom correspondence should be addressed.

Tel: +3225556245; Fax: +3225556257; Email: maud.dedieuleveult@gmail.com, rdeplus@ulb.ac.be and ffuks@ulb.ac.be 


\section{ABSTRACT}

TET proteins convert 5-methylcytosine $(5 \mathrm{mC})$ to 5-hydroxymethylcytosine $(5 \mathrm{hmC})$, leading to a dynamic epigenetic state of DNA that can influence transcription. While TET proteins have been associated with either epigenetic repression or activation complexes, the overall understanding of the molecular mechanisms involved in TETmediated regulation of gene transcription still remains limited. Here, we show that TET proteins interact with lymphoid-specific helicase (LSH), a chromatin remodeling factor belonging to the SNF2 super family. Lsh knock-out leads to a significant reduction of 5-hydroxymethylation global level in mouse embryonic fibroblasts (MEFs) and in embryonic stem cells (ESC). Whole genome sequencing of $5 \mathrm{hmC}$ in wild-type versus Lsh knock-out MEFs and ESCs showed that in absence of Lsh, some regions of the genome gain $5 \mathrm{hmC}$ while others lose it, with not much effect on gene expression. We further show that $5 \mathrm{hmC}$ modifications upon Lsh loss is not a direct consequence of $5 \mathrm{mC}$ decrease, as differentially hydroxymethylated regions (DhMR) did not overlap with DMR (differentially methylated regions), underlying that these modifications occurred at different genomic loci. Altogether, our results suggest that $\mathrm{LSH}$ is a key regulator of $5 \mathrm{hmC}$ in both MEFs and ESC and that TET proteins rely on specific factors to establish genome-wide $5 \mathrm{hmC}$ patterns. 


\section{INTRODUCTION}

DNA modifications and chromatin organization play a major role in a variety of biological and molecular processes. The best known modification base in DNA is 5methylcytosine $(5 \mathrm{mC})$ established by the DNA methyltransferases (DNMTs) $(1,2)$. DNA methylation contributes to numerous biological processes, including embryonic development, X-chromosome inactivation, genomic imprinting and chromosome stability (3). The discovery of TET proteins, 2-oxyglutarate and $\mathrm{Fe}(\mathrm{II})$-dependent dioxygenases which are able to oxidize $5 \mathrm{mC}$ in 5-hydroxymethylcytosine, 5formylcytosine $(5 f C)$ and 5-carboxylcytosine $(5 \mathrm{caC})$, has revolutionized our understanding of DNA demethylation process (4-6). In addition to being an intermediate in DNA demethylation, $5 \mathrm{hmC}$ is now established as an epigenetic mark and has been shown to be the mostly stable in the mammalian genome $(7,8)$. New readers and modifiers are thus rising interest in getting deeper into the roles and the dynamics of $5 \mathrm{hmC}$ (9). Despite substantial research describing the genomic landscape of $5 \mathrm{hmC}$ in different cell systems, our understanding of the TETs and $5 \mathrm{hmC}$ regulation remains limited.

Approaches to elucidate how TET proteins function included search for interacting partners and led to the identification of transcriptional factors /nuclear receptors (CXXC4, NANOG, PPARY, PU.1, EBF1, PRDM14), chromatin-associated proteins involved in transcriptional activation (OGT and SET1/COMPASS complex) (10) or repression (SIN3A/HDACs, NURD) (11). Also, insights have been obtained regarding the regulation of TET activity and expression. Ascorbic acid (vitamin $\mathrm{C}$ ) has been shown to significantly enhance TET hydroxymethylation activity, acting as a specific co-factor for these enzymes probably by reducing $\mathrm{Fe}$ (III) back to $\mathrm{Fe}$ (II) after enzymatic catalysis (12-15). Tet1 can be regulated by several transcription factors as NF-kB with an anticorrelation with immune response genes in various cancer cell lines (16). Other groups indicated that TET proteins can be post-transcriptionally regulated by microRNAs (miRNAs) (17-20). Altogether, these results shed lights on regulatory mechanisms (post-translational modifications, miRNA network, small molecules) that impact TET expression and/or activity.

Various cancers and transformed cells harbor disturbed epigenomes, as illustrated by global DNA hypomethylation and focal promoter hypermethylation (21, 
22). Interestingly, TET2 and IDH1/IDH2 have been found to be mutated in different cancers, including myeloproliferative neoplasms (MPNs), myelodysplastic syndromes (MDS) and acute myeloid leukemia (AML) (23-25). The regulation of TET proteins expression, without implying specific mutations, has also been involved in the development of cancers, for instance TET2 expression was significantly reduced in melanoma, causing global hypo-hydroxymethylation and hypermethylation of genes involved in disease progression (26). Although decreased $5 \mathrm{hmC}$ seems to be a common feature of various cancers, the precise mechanism linking TET deregulation and aberrant methylation is not properly understood yet.

The SNF2-like helicase LSH (also known as HELLS, SMARCA6 or PASG), initially identified as a factor required for lymphoid cells proliferation, belongs to the SNF2 family of chromatin remodeling factors $(27,28)$. Chromatin remodelers play important biological functions, such as transcriptional control, DNA repair and genomic recombination (29). LSH was shown to be able to slide nucleosomes in vitro $(30,31)$. Deletion of $L s h$ in mice was reported to be perinatally lethal with normal development, except for a small birth weight (32). Connection between Lsh and DNA methylation is well established. Its mutation or deletion leads to a profound loss of global DNA methylation, especially at repetitive sequences $(33,34)$ but also leads to hypo- as well as hypermethylation at specific genes (35). Knockdown of Lsh in embryonal carcinoma cells and ES cells, despite incomplete CG methylation, Lsh-/embryos show cellular differentiation (36). In addition to reduced DNA methylation levels, the chromatin of Lsh-/-MEFs displays alterations in histone modifications, such as H3K4me3, H3K27me3 and H3K9me3 $(37,38)$.

Mechanistically, LSH acts as a recruitment platform for DNMTs, as it has been shown to interact with DNMT3A and DNMT3B and indirectly with DNMT1 and also CDCA7 $(31,39,40)$. LSH may facilitate DNMTs recruitment by remodeling chromatin at their target genes. More recently, the ATP function of LSH was shown to be critical for nucleosome density and for complete cytosine methylation at specific loci, thereby implying that chromatin remodeling mediated by LSH is critical for the establishment of DNA methylation patterns (41). However, it is still unclear whether LSH loss affects the maintenance of DNA methylation patterns in addition to playing a role in de novo methylation $(36,39,42,43)$, when during development these effects occur and if other factors (apart from DNMTs) are involved in this process. Recently, new work 
has been done to understand the crosstalk between the two roles of LSH on methylation and nucleosome positioning suggesting that LSH is needed at enhancers and repetitive sequences to regulate the chromatin accessibility $(41,44)$.

In this study, we showed that LSH and TETs interact. We found that Lsh disruption leads to a reduction in the global level of $5 \mathrm{hmC}$ in MEFs and ESCs, which are well studied models for both TET and LSH. Nonetheless, genome-wide 5hmC studies in wild-type and Lsh"- MEFs and ESCs revealed that thousands of genomic regions gain or lose $5 \mathrm{hmC}$. In most cases no changes in gene expression could be detected. Our data showed that $5 \mathrm{hmC}$ modifications upon Lsh loss were not a direct consequence of changes in $5 \mathrm{mC}$ in these cells. Altogether, we identified the SNF2like helicase LSH as a regulator of the hydroxymethylome at the genome-wide level. 


\section{MATERIALS AND METHODS}

\section{Cell Culture, transient transfection and Infection}

Wild-type and Lsh KO MEFs (kindly received from Dr K. Muegge, (39)) were cultured in Dulbecco's modified Eagle's medium (DMEM) supplemented with 10\% FBS and $100 \mathrm{U} / \mathrm{ml}$ of penicillin/streptomycin. All cells were grown at $37^{\circ} \mathrm{C}$ in an atmosphere of $5 \% \mathrm{CO}_{2}$. Wild-type, Lsh ${ }^{\text {off/off }}$ mouse embryonic stem cells (by $\mathrm{Dr}$. Stancheva) were expanded on feeders using regular ES media (DMEM supplemented with 15\% FBS, penicillin/streptomycin, non-essential amino acids, $1 \mathrm{mM}$ sodium pyruvate, $2 \mathrm{mM}$ L-glutamine and $100 \mathrm{nM}$ of $\beta$-mercaptoethanol) containing leukemia inhibitory factor (LIF). A modified knock-in strategy and allele design previously reported by Schnütgen et al (45) were employed to generate the Lsh ${ }^{\text {off/off }}$ ES cells by sequential targeted disruption of both Lsh alleles in E14 (129/Ola) ES cells. We first integrated by homologous recombination a reversible stop cassette (SA-GFP-Neo) flanked by a set of LoxP and Frt sites into the third intron of the Lsh gene. The integrated stop cassette is predicted to generate a null $L$ sh allele, which we named $L s h^{\text {off }}$, producing a chimeric protein containing 72 amino acids of the LSH N-terminus, which lacks nuclear localisation signal and any known function, fused to a GFP-Neomycin marker. The second Lsh allele was disrupted in one of the $L s h^{\text {off/ }+}$ ES cell lines by targeted integration of the same stop cassette, but this time carrying a hygromycin resistance marker. The successful integration of both stop cassettes was confirmed by Southern and Western blots. We will further refer to these Lsh ${ }^{\text {off/off }}$ ES cells as Lsh KO ESCs.

Transient transfections were performed as described (46). Knock down in ES cells were designed accordingly to the authors' instructions and performed in V6.5 ESCs (kindly received from Dr K. Koh, KU Leven) (47). Sequences used are KD1: CCGGCTAATCAGGGAGTTAAA, KD2: TCGAATGCTGCCCGAACTTAA, KD3: GGACACAGGATTAAGAATATG. Retrovirus production by HEK293GP cells and infection of target cells were performed as described (46). Infected cells were selected with $1.5 \mu \mathrm{g} / \mathrm{ml}$ puromycin (Sigma).

\section{Halo Tag (HT) mammalian pulldown assay}

HT mammalian pulldown assays were performed as previously described (10). Briefly, HEK293T cells were transfected with Halotag plasmid. Cells expressing HT- 
fusion proteins or HT-Ctrl were incubated in the mammalian lysis buffer supplemented with Protease Inhibitor cocktail (Promega) and RQ1 RNase-Free DNase (Promega) for 10min on ice.

The clarified lysate was incubated with HaloLink Resin (Promega) for 15min at $22^{\circ} \mathrm{C}$ with rotation. The resin was then washed with wash buffer and protein interactors were eluted with SDS elution buffer. Affinity purified complexes were then analyzed by nano-LC/MS/MS (MSBioworks) and Western blotting.

\section{Immunoprecipitation assays}

Whole-cell extracts were prepared in IPH lysis buffer $(50 \mathrm{mM}$ Tris- $\mathrm{HCl} \mathrm{pH} 8$, $150 \mathrm{mM} \mathrm{NaCl}, 5 \mathrm{mM}$ EDTA, 0.5\% NP40 supplemented with protease inhibitor cocktail (Roche). Immunoprecipitations were performed with anti-rabbit or anti-mouse IgG Dynabeads $\AA$ (Life Technologies) overnight at $4^{\circ} \mathrm{C}$. The primary antibodies used in these experiments were directed against the following: control immunoglobulin $G$ (lgG) (rabbit sc-2027 and mouse sc-2025; Santa Cruz), FLAG M2 (F3165; Sigma), LSH (NB100-278; Novus), TET1 (09-872; Millipore) and TET2 (R1086-4; Abiocode). The immunoprecipitated complexes were eluted in Laemmli buffer.

\section{GST pulldown assays}

Full-length Lsh, Lsh CC (aa 1-226), Lsh DEXD (227- 589) and Lsh CT (590838) domains cloned in the pGEX4T-1 vector were kindly provided by $\mathrm{Dr} \mathrm{U}$. Ziebold (Cancer Research, Berlin, Germany) (48). Recombinant GST-fused proteins were expressed in and purified from Escherichia coli BL21, as previously described. We performed in vitro transcription/ translation using the TNT system (Promega) with pcDNA3 expression vectors for murine Flag-tagged Tet catalytic domains or fulllength Halo-tagged human Tet proteins. GST-pulldown assays were performed as previously described (49).

\section{Dot blot}

Genomic DNA was extracted with the DNeasy blood and tissue kit (Qiagen), denatured for $10 \mathrm{~min}$ at $95^{\circ} \mathrm{C}$, chilled directly on ice and spotted on a nylon membrane (GE Healthcare Hybond-N ${ }^{+}$). After UV-induced fixation of target nucleic acids ( $2 \times 200000 \mu \mathrm{J} / \mathrm{cm}^{2}$ of UV), the membrane was blocked in PBS BSA $1 \%$ and incubated with anti-5hmC (39769; Active Motif) or anti-ssDNA antibody (LS-C64821; LSBio). 


\section{Analysis of global DNA 5mC and 5hmC levels by mass spectrometry (LC- MS/MS)}

Analysis of global DNA $5 \mathrm{mC}$ and $5 \mathrm{hmC}$ levels by LC-MS/MS was carried out as described in Bachman et al (8). Briefly, $500 \mathrm{ng}$ of genomic DNA was incubated with $5 \mathrm{U}$ of DNA Degradase Plus (Zymo Research) at $37^{\circ} \mathrm{C}$ for $3 \mathrm{~h}$. The resulting mixture of 2'-deoxynucleosides was analysed on a Triple Quad 6500 mass spectrometer (AB Sciex) fitted with an Infinity 1290 LC system (Agilent) and an Acquity UPLC HSS T3 column (Waters), using a gradient of water and acetonitrile with $0.1 \%$ formic acid. External calibration was performed using synthetic standards, and for accurate quantification, all samples and standards were spiked with isotopically labeled nucleosides. $5 \mathrm{mC}$ and $5 \mathrm{hmC}$ levels are expressed as a percentage of total cytosines $(\mathrm{C}, 5 \mathrm{mC}$ and $5 \mathrm{hmC})$.

\section{hMeDIP}

$1 \mu \mathrm{g}$ of genomic DNA was diluted in ultra-pure water to $35 \mathrm{ng} / \mu \mathrm{L}$ and then sonicated in cold water with a Bioruptor sonicator (Diagenode) to obtain fragments averaging $300 \mathrm{bp}$ in size. The fragmented DNA was used in combination with the hydroxymethyl collector (Active Motif) following the manufacturer's protocol. Briefly, a glucose moiety that contains a reactive azide group was enzymatically linked to hydroxymethylcytosine in DNA, creating glucosyl-hydroxymethylcytosine. Next, a biotin conjugate was chemically attached to the modified glucose via a "click reaction", and magnetic streptavidin beads were used to capture the biotinylatedhmC DNA fragments. After extensive washing steps and chemical elution, the hydroxymethylated DNA fragments released from the beads were used in sequencing experiments.

\section{Library preparation, deep sequencing workflow and data analyses}

The library preparation was performed using the TruSeq ChIP Sample Prep Kit (Illumina). Briefly, double stranded DNA was subjected to 5' and 3' protruding ends repair and non-templated adenines were added to the 3' ends of the blunted DNA fragments to allow ligation of multiplex Illumina's adapters. DNA fragments were then size selected (300-500bp) in order to remove all non-ligated adapters. 18 cycles of PCR were done to amplify the library which was then quantified by fluorometry 
using the Qubit 2.0 and its integrity was assessed with 2100 bioanalyzer (Agilent) before being sequenced. 6pM of DNA library, spiked with $1 \%$ PhiX viral DNA, were clustered on cBot (Illumina) and sequencing was performed on a HiScanSQ module (Illumina). The BWA software was used to map sequencing reads to the mouse genome (NCBI Build 37/ UCSC mm9). Reads not uniquely mapped to the reference genome were discarded. Read density was computed by removing duplicate reads and by normalizing the local read count with respect to the total read count. Local enrichment in $5 \mathrm{hmC}$ was evaluated with the MACS software with a p-value cut-off < 10e-10. Differences between 5hmC levels were assessed for all annotated RefSeq transcripts (Gene Body) by first computing the normalized number of reads and then the fold change and absolute delta value between the two conditions. Genes were sorted according to their absolute fold change after discarding genes with a low absolute difference to avoid artefacts. $5 \mathrm{hmC}$ profiles around the TSS and within exons and introns were computed by first computing the local enrichment with the MACS software and then annotating the enrichment with the CEAS software. All statistics and plotting were done with the $\mathrm{R}$ statistical software. To obtain sequencing tracks, bedGraph files (genomeCoverageBed) were uploaded onto the IGV genome browser.

\section{Bioinformatic analysis}

To identify the differentially hydroxymethylated regions, the genome was first structured in fixed windows (5000bp). The normalized $5 \mathrm{hmC}$ levels were then estimated by computing the FPKM for every window and each condition. The regions (windows of 5000bp) were then ranked based on their fold change and relative difference. Regions with an absolute fold change $>2$ and absolute difference of at least 1 were selected for downstream analysis. Regions were related to genomic features using the VISTA Enhancer database (https://enhancer.lbl.gov), UCSC RefSeq and $\mathrm{CpG}$ islands annotations and by computing the genomic overlap between the region center and those features. The genomic regions between the transcription start site (TSS) and the transcription termination site (TTS) as defined as Gene Body, the $2 \mathrm{~kb}$ regions upstream the TSS was defined as the promoter. Regions ambiguously overlapping multiple features were associated with multiple categories. For the metagene analysis all the RefSeq genes were used to compute the relative average $5 \mathrm{hmC}$ signal inside the transcript (TSS to TTS). 
Public databases have been used: Histones marks ChIP-seq (GSE90893). RNA-seq data from ES cells GSM1581307 was used. Transcriptomic analyses for MEFs Lsh KO are downloaded from E-MEXP-2383 and methylation array from EMEXP-2385(43). hMeDIP-seq on WT and Lsh KO MEFs and ES cells were uploaded under the GSE110129 number (token number: sfgrcoswrtybxej).

For the comparative analysis, ChIP-seq of histones marks were downloaded from GSE90893 and aligned onto the mm9 genome. Occupancy of 7 histones marks and one histone variant on the DhMR were then visualized with the seqMINER software(50). Free clustering was done and the count in each category with specific histone profile was performed.

\section{Repetitive element analysis (Pseudogenome)}

A pseudogenome was generated with mouse DNA repeats sequences from RepeatMasker. Reads from fastq were mapped on this pseudogenome, using bowtie allowing two mismatches and without keeping reads mapped to more than one site. Duplicated reads were removed using samtools, and total reads mapped to each DNA repeats were calculated using samtools. The total numbers of reads mapped to each repeat element were normalized to the number of reads sequenced for each sample. To assess the effective change between the Lsh KO and the WT cells, the log odds ratio and P-value using a Fisher exact test were computed for each repetitive element. The $\mathrm{P}$-values were then corrected using the FDR correction method.

\section{$R T-q P C R$ and Gene expression}

Total RNA was extracted with the RNeasy Mini kit (Qiagen). After DNase I treatment (DNA-free DNase kit, Ambion), Superscript II reverse transcriptase (Invitrogen) were used to reverse-transcribe mRNAs to cDNAs. Gene expression levels were then evaluated by real-time PCR (LightCycler 480, Roche). Primers used are available upon request.

\section{Ingenuity software.}

Ingenuity IPA software was used to identify the top molecular and cellular functions. The genes and regions lists were loaded into the Ingenuity database and then core analyses were done, using default parameters. 
bioRxiv preprint doi: https://doi.org/10.1101/2020.03.10.983148; this version posted March 11, 2020. The copyright holder for this preprint (which was not certified by peer review) is the author/funder, who has granted bioRxiv a license to display the preprint in perpetuity. It is made available under aCC-BY-NC-ND 4.0 International license. 


\section{RESULTS}

\section{LSH is a TET-interacting factor}

To explore the mechanisms of action of TETs, we identified TET protein partners via an unbiased proteomic approach using HaloTag technology as previously described (51). This approach identified several transcription factors and epigenetic proteins co-immunoprecipitating with TET proteins, including previously described binding partner OGT (10), PARP (52) and PCNA (53). We also identified the SNF2-helicase like LSH as a TET1 and TET3 binding partner with this method (data not shown).

To further explore the interaction between TETs and LSH, we first confirmed by semi-endogenous and endogenous co-IPs their interaction. As shown in Figure 1A, when HEK293T cells were transfected with Flag-tagged TET1, TET2 or TET3 catalytic domain (CD) and the empty vector. We found by immunoprecipitation that all TET CDs to interact with endogenous LSH (right, top panel, lane 6-8). LSH was not detected in the immunoprecipitated complex after transfection of cells with the Flagempty plasmid (right, top panel, lane 5). Controls showing that the protein of interest was correctly immunoprecipitated (right, bottom panel) and that all proteins were expressed in cell lysates (left panel) are shown. These results were further confirmed in HEK293T cell lysates transfected with full-length Halo-tagged TET proteins (data not shown). The reverse IP was next performed (Figure S1A) confirming the interaction between LSH and TET proteins.

To map the LSH domain interacting with TET proteins, we performed additional GST pulldown assays. As shown in Figure S1B TET proteins were found to interact in vitro via their catalytic domain with the coiled-coil domain (CC) of LSH which is known to interact with DMNT1 and DNMT3B $(48,54)$.

To further validate the interaction between TET and $\mathrm{LSH}$, we performed endogenous co-IPs in mouse embryonic stem cells using antibodies specific for LSH (Figure 1B) and for TET1 (left panel) or TET2 (right panel). We did not assess for TET3/LSH interaction in ES cells because TET3 is expressed at low levels in this cell system. Our results indicated endogenous interactions in ESC between LSH and TET1, and between LSH and TET2. Our data indicate that LSH and TET proteins interact in vitro and in vivo. 


\section{Lsh knock-out impairs the global hydroxymethylation levels in MEFs and ES cells}

Previous studies have shown that Lsh is involved in the establishment and maintenance of $5 \mathrm{mC}(35,36,41)$. Since DNA methylation is the substrate of hydroxymethylation, we thus decided to test whether Lsh could also contribute to $5 \mathrm{hmC}$. To explore the role of Lsh in $5 \mathrm{hmC}$, we performed dot blot experiments and mass spectrometry (MS) analyses (Figure 2). We prepared genomic DNA samples from Lsh KO and WT ES cells and analyzed them by dot blot with anti-5hmC and anti-single stranded DNA antibodies as a control. The quantification of the images by ImageJ showed that the levels of $5 \mathrm{hmC}$ are lower in Lsh $\mathrm{KO}$ compare to WT ES (Figure 2A). We reanalyzed the same samples by MS and confirmed the lower levels (29\%) of $5 \mathrm{hmC}$ in the absence of Lsh without affecting the levels of $5 \mathrm{mC}$ (Figure $2 \mathrm{~B}$ ). We validated these observations by showing that depletion of Lsh by short-hairpin RNA (shRNA) in ES also causes a 50\% reduction (shRNA 1), 32\% (shRNA 2) and $70 \%$ (shRNA 3 ) of the $5 \mathrm{hmC}$ levels, which is similar to the $40 \%$ reduction observed by dot blot in KO ES cells (Figure S2 panel A and C). We also observed that another mark of demethylation, the 5-formylcytosine $(5 \mathrm{fC})$, is also dramatically reduced by 15 fold (Figure S2 panel B). Surprisingly, we observed that the levels of $5 \mathrm{mC}$ were similar between Lsh $\mathrm{KO}$ and control ES at global level, suggesting that Lsh loss directly reduces $5 \mathrm{hmC}$ establishment and/or maintenance rather than by an influence on DNA methylation.

Our preliminary MS data showed that the levels of $5 \mathrm{hmC}$ are 10 times more abundant in ES than in MEFs cells. Nevertheless, we observed by dot blot that $5 \mathrm{hmC}$ levels were 1/3 lower in Lsh KO MEFs compared to WT MEFs (Figure 2C). Again, MS confirmed this result by showing a $63 \%$ reduction of $5 \mathrm{hmC}$ (Figure 2D). In contrast to what we detected in ES, we observed a reduction (by $40 \%$ at the genomewide level) in the levels of $5 \mathrm{mC}$ by MS in the absence of Lsh as previously described $(36,43,55)$.

Altogether, our results reveal that LSH maintain and/or establish 5hmC levels in ES and MEF cells. In ES cells, we could not detect significant changes in $5 \mathrm{mC}$ in the absence of Lsh suggesting that in this particular cell type Lsh could directly affect the function of Tet enzymes and 5hmC patterns. On the contrary, in MEFs cells, Lsh 
regulates both $5 \mathrm{mC}$ and $5 \mathrm{hmC}$ levels. Lsh may thus be involved in the regulation of $5 \mathrm{hmC}$ patterns in different ways according to the cell type.

\section{5hmC changes at the genome-wide level in Lsh KO ESCs, mostly in gene bodies.}

To further explore the role of LSH in the distribution of $5 \mathrm{hmC}$, we performed a genome-wide mapping of hydroxymethylation (hMeDIP-seq) and compared Lsh KO ESCs and MEFs Lsh KO to their wild-type counterparts (Figure 3 and Figure 4). Sequencing reads from Lsh KO and WT sample were aligned to the mouse $\mathrm{mm} 9$ genome. Using a window-based approach (5,000bp), we analyzed the read density along chromosomes and compared the density in Lsh KO and WT cells (cf Methods).

In Lsh KO ESCs, we identified 8557 windows (down 4312, up 4245, no statistical difference), corresponding to 5017 genes, showing differential $5 \mathrm{hmC}$ levels in ES cells, as shown for the representative gene Podxl2 (Figure 3C). Half of these regions show gain and loss in 5hmC (Figure 3A). We found 2659 genes with gain of $5 \mathrm{hmC}$ and 2448 genes with loss of $5 \mathrm{hmC}$ (Supplemental Table 1). A deeper analysis of the distribution of these regions show enrichment at promoters (124/96 expected), gene bodies (4801/2940 expected), enhancers (13/5), multiple regions (i.e. region with at least two different genomic features, e.g. promoter and enhancer) (830/395 expected) and an underepresentation of intergenic regions (2789/5122 expected). These data indicate that regions with significant changes in $5 \mathrm{hmC}$ are mainly present in gene bodies and rarely occur at intergenic regions (Figure 3B). A functional analysis of hyper- and hypo- hydroxymethylated genes revealed significant overrepresentation of pathways associated with development, such as "tissue development", "embryonic development" and "organismal development" (Figure S3D). We validated the changes in $5 \mathrm{hmC}$ profile by hMeDIP-qPCR. We confirmed that $5 \mathrm{hmC}$ was increased at Sc/36, GM5122, Zfat and Alox15 loci, while it was reduced at Mf151, Hspa, Mef2d and Gbj5 (Figure 3D), as observed in hMeDIP-seq. No change of $5 \mathrm{hmC}$ was detected at two different control regions (Figure 3D). 
$5 \mathrm{hmC}$ changes often occur at gene bodies (>55\%, Figure 3B). In contrast, significant changes in $5 \mathrm{hmC}$ rarely occur at promoters, but still in a higher than expected proportion ( $<3 \%$ of cases, Figure $3 \mathrm{~B}$ ), and at $\mathrm{CpG}$ islands (Figure S3A).

We investigate the $5 \mathrm{hmC}$ levels on the gene showing loss and gain of $5 \mathrm{hmC}$ upon LSH KO in the WT condition with Metagene analysis (Figure S3B). This analysis showed that both categories of genes have a higher level in 5hmC compared to the entire set of mouse genes. Nonetheless, no difference between the $5 \mathrm{hmC}$ level between hyper- and hypo- hydroxymethylated genes at the gene scale. A more detailed view of gene promoters showed a slight accumulation of $5 \mathrm{hmC} 1 \mathrm{~kb}$ upstream to the TSS for hyper-hydroxymethylated genes (Figure S3C).

\section{5hmC changes at the genome-wide level in Lsh KO MEFs.}

In Lsh KO MEFs, we identified 9002 tilling regions, corresponding to 3138 genes with differential hydroxymethylation. $77 \%$ of the regions $(n=6932)$ showed hypo-hydroxymethylation and $23 \%$ of the regions $(n=2070)$ harbor hyperhydroxymethylation (Figure 4A) corresponding to 2376 and 1043 genes, respectively. As previously observed in ES cells (Figure 3B), these regions are mostly found in gene bodies (Figure 4B). Also, hydroxymethylation changes are rare at promoters (Figure 4B) and far from CpG islands (Figure S4A). No significant pattern is observed between genes hyper- and hypo-hydroxymethylated at TSS, gene body and TTS (Figure S4B). Changes in $5 \mathrm{hmC}$ level detected by high throughput sequencing were further confirmed by qPCR. Bdnf, Btg4, Elfn2 and Fam92 harbored an increased $5 \mathrm{hmC}$ whereas Hp, Gdf5, Klf2 and Sfi1 showed a decrease of $5 \mathrm{hmC}$ as in hMeDIPseq (Figure 4C-D). No change of $5 \mathrm{hmC}$ was detected at control regions 1 and 2 (Figure 4D). Functional analysis of differentially hydroxymethylated genes showed enrichment in GO term "tissue development", "tissue morphology" and "organismal development" (Figure S4C) highlighting again the global role of Lsh and the implication of (hydroxy)methylation in global development $(41,56,57)$.

\section{Lsh regulates $5 \mathrm{hmC}$ at repetitive sequences.}

Lsh KO MEFs cells have been previously characterized by different groups $(32,55,58,59)$. A common finding is the global loss of $5 \mathrm{mC}$, including at repeated 
minor satellite sequences. We directly accessed the level of $5 \mathrm{hmC}$ at major and minor satellite as well as repeated sequences such as LINE1 and SINE1 (Figure S5A). We observed by hMeDIP-qPCR a mild decrease in 5hmC at minor satellites and LINE1 elements in Lsh MEF KO cells, while a slightly decrease is detected at major satellite and a weak increase observe at SINE elements. We further expended the analysis to all DNA retreated sequences in the mice genome (Figure S5B). We mapped the reads on a synthetic pseudogenome containing all the repeated elements (Figure S5B) and identified several classes and families of repeated elements that exhibit changes in hydroxymethylation between Lsh KO MEFs and controls. In some cases, we observed loss of $5 \mathrm{hmC}$ at these repeated sequences. We next performed the same analysis in ES cells and found that major and minor satellites were hypo- hydroxymethylated. Thus, besides gene-regions, Lsh seems to influence hydroxymethylation of some repetitive sequences in ES and MEF cells.

\section{Relationship between 5hmC, $\mathrm{mC}$ and gene expression in MEF cells}

In Lsh KO MEF cells, a common finding is the global loss of $5 \mathrm{mC}$, including at repeated minor satellite sequences $(34,60)$. Myant and colleagues performed a genome-wide description of the global demethylation on Lsh KO MEFs (43). We used this array (RefSeq promoter microarray) to investigate the role of Lsh on $5 \mathrm{mC}$ and $5 \mathrm{hmC}$ pattern.

We first compared regions harboring deregulated methylation and hydroxymethylation in MEFs cells. We found only $17 \%$ of overlap and the overlap was not statistically significant (Figure 5A), which suggested that the patterns of DMRs and DhMRs were different. For instance, 5hmC changes observed at specific sites (Figure 4D), were not associated with changes in $5 \mathrm{mC}$ (Figure 5B). These findings suggest that $5 \mathrm{mC}$ and $5 \mathrm{hmC}$ patterns are not directly related to each other in MEF cells.

Myant and colleagues performed a global analysis of the transcriptionally misregulated genes in Lsh KO versus WT MEFs cells (43). We used these data to compare the regions showing differential $5 \mathrm{hmC}$ with expression data. We found (Figure $5 \mathrm{C}$ ) that only $15 \%$ of genes that harbor DhMRs also have alteration of gene expression and $16 \%$ of genes that harbor differential expression have alteration of $5 \mathrm{hmC}$ level and the overlap was not significant. Also, no global expression changes 
were found between gene with deregulated $5 \mathrm{hmC}$ to all others (same result was obtained with hypo- and hyper-hydroxymethylated genes separately) (Figure S6). Thus, we confirmed that at the genome-wide level, changes in hydroxymethylation landscape around genes only partially affect their expression. Previous papers described also a poor overlap ( 10\%) between loss or gain of DNA methylation and gene expression changes (43).

In order to understand deeper the epigenetic status of the identified DhMRs, we next compared the 9002 DhMRs identified in MEFs cells to different histones marks that are characteristics of different chromatin environment or processes such as promoters (H3K4me3 and $\mathrm{H} 3 \mathrm{~K} 9 \mathrm{Ac})$, active promoters and enhancers (H3K4me2 and $\mathrm{H} 3 \mathrm{~K} 27 \mathrm{Ac}$ ), transcription (H3K36me3 and $\mathrm{H} 3 \mathrm{~K} 79 \mathrm{me} 2)$ or repressive compartments (H3K27me3) (61) (GSE90893) (Figure 5D). 21\% of the DhMRs correlated with active histones marks: H3K27Ac, H3K4me2/3, H3K9Ac (group 1 and 2), 19\% correlated with elongating marks as H3K36me3 and H3K79me2 (group 3 and 4 ) and only a few (10\%) with repressive marks, H3K27me3 (group 5). For group 6 , we found that half of the DhMRs do not harbor any examined marks. These data suggest the Lsh driven $5 \mathrm{hmC}$ is linked to different specific chromatin state and regions. 


\section{DISCUSSION}

$\mathrm{LSH}$, a chromatin remodeler, was linked to DNA methylation and already known to interact with DNMTs $(39,40)$ and influence DNA methylation, in particular at repetitive sequences $(33,62)$. Here, we show that LSH is also able to interact with the DNA hydroxymethylases, the TET proteins. LSH binds the catalytic domain of TETs. On the other hand, TETs interact with the CC domain of LSH. CC domains are often involved in protein-protein interaction and this region of $\mathrm{LSH}$ is required for transcriptional silencing independently of its chromatin-remodeling activity (40). TETs and DNMTs both interact with the CC domain of LSH. Additional experiments are needed to answer the question of whether the binding of TETs and DNMTs to LSH is mutually exclusive.

The landscapes of $5 \mathrm{hmC}$ have been described for numerous cell type from human and mouse origin, but the molecular mechanisms involved in the deposition, maintenance and regulation of this epigenetic mark still remains unclear. Several studies have been performed to better understand the dynamics of $5 \mathrm{hmC}$ upon differentiation and cell growth changes (63-66). In addition, studies have been designed to identify the factors involved in $5 \mathrm{hmC}$ deposition and binding $(11,67,68)$. Among these studies, one reported that LSH binds $5 \mathrm{hmC}$ containing DNAs in vitro, while LSH does not bind to the same oligonucleotide containing $5 \mathrm{mC}(9)$. It was also previously described that LSH can also induce the expression of TET enzymes (69). Then, LSH could modulate the $5 \mathrm{hmC}$ by increasing Tet mRNA level and/or influence their catalytic activity by interacting with $5 \mathrm{hmC}$ writers $(9,69)$. To decipher the exact contribution of each mechanism, producing a mutant form of Lsh that is unable to interact with TETs would be necessary.

In dot-blot, MS and deep sequencing experiments, we observed that the patterns of $5 \mathrm{hmC}$ were globally altered in ESCs and MEFs lacking LSH. These alterations in $5 \mathrm{hmC}$ are both gain and loss of $5 \mathrm{hmC}$ at specific genomic sites and repeated DNA sequences. Previous studies have shown a role of Lsh in $5 \mathrm{mC}$ in MEF cells. Comparison with published data (43) provides evidence that most changes of $5 \mathrm{mC}$ and $5 \mathrm{hmC}$ do not occur at the same genomic regions. Moreover, regions with $5 \mathrm{mC}$ changes do not correspond to changes of $5 \mathrm{hmC}$. This is further consistent with a direct role of Lsh in establishment and/or maintenance of $5 \mathrm{hmC}$ pattern in ES and MEF cells. Unexpectedly, our results for the first time reveal a $5 \mathrm{hmC}$ regulation 
independently of $5 \mathrm{mC}$ patterns. The use of an auxin-inducible degron Lsh model could help to decipher precisely the dynamic of Lsh influence on ESCs and MEFs epigenomes.

Nevertheless, LSH seems to be a central regulator of DNA epigenome by influencing not only DNA methylation but also DNA hydroxymethylation. In particular, LSH can influence $5 \mathrm{hmC}$ by different mechanisms: (i) by interacting with TETs and potentially influencing their catalytic activity, (ii) by increasing Tets expression (70) and (iii) by binding to hydroxymethylated DNA (9).

The ES cells lacking LSH are viable and remain pluripotent indicating that changes in $5 \mathrm{hmC}$ and gene expression induced by Lsh $\mathrm{KO}$ affect, only modestly, the biology of ES cells. We postulate that changes in $5 \mathrm{hmC}$ levels upon Lsh $\mathrm{KO}$ are not sufficient to completely disorganize the transcriptional network of ES cells. 5hmC changes driven by Lsh KO are not associated with specific histone mark. However, $5 \mathrm{hmC}$ could generate a primed and more permissive state that could facilitate future transcriptional induction. It would be interesting to further investigate the dynamic of $5 \mathrm{hmC}$ and gene expression when Lsh KO ESCs differentiate into different lineages (ectoderm, mesoderm and endoderm). The functional analysis of genes affected by Lsh $\mathrm{KO}$ indicates that most of them are involved in development. Changes in 5hmC at specific genes in ES cells might be linked to changes in gene expression during specific steps of cell differentiation. Furthermore, it is also possible that LSH plays a broader function in genome organization beyond the local nucleosome remodeling activity. We observed that several families of DNA repeats exhibit changes in 5hmC upon Lsh KO in ES and MEF cells. It was also observed that the chromatin structure of repetitive sequences is impaired upon Lsh knock down $(33,71)$. These changes might contribute to the organization of specific chromosomal domains in the nucleus, linked to changes in epigenetic landscape and maybe gene expression in specific conditions. Further exploration is needed to uncover such mechanism.

LSH is located in a break point region frequently associated with leukemia (27) and a deletion in LSH gene is found in $57 \%$ of AML and $37 \%$ of ALL (72). Interestingly, $5 \mathrm{hmC}$ is often deregulated in hematological malignancies and TET2 is one of the most mutated genes in leukemia. In mice, loss of Tet2 increases the hematopoietic stem cell compartment and skews cell differentiation towards the 
myeloid compartment $(73,74)$. Both $5 \mathrm{hmC}$ levels and $\mathrm{LSH}$ are reduced in several solid tumors as nasopharyngeal carcinoma, breast or colon cancer (70). The link between LSH, TETs and $5 \mathrm{hmC}$ is not yet explored in hematopoietic diseases and further investigation could open new therapeutic possibilities.

In summary, we report here an interaction between TETs and LSH. Our genome-wide $5 \mathrm{hmC}$ profiles in MEFs and ES Lsh KO mouse cell lines suggest that LSH is an important regulator of DNA hydroxymethylation. Many efforts had been invested in studying the role of LSH in DNA methylation and little has been done on nucleosome positioning despite the classification of LSH as a full member of SNF2 ATPase remodeler family. Related to this function, we strongly suggest here a genome wide crosstalk between nucleosome positioning/chromatin organization and hydroxymethylation, which must be explored further. 


\section{ACKNOWLEDGMENT}

We thank Dr K. Muegge for the generous gift of MEFs Lsh WT and KO. We thank Dr M. Defrance for bioinformatical analyses and Dr C. Marchal for the technical help for repetitive region analysis. We thank $\mathrm{Dr} U$. Ziebold for the generous gift of Lsh plasmids. We thank Dr B. Miotto for helpful discussions.

\section{AUTHORS CONTRIBUTIONS}

MdD, RD and FF designed and coordinated the study. MdD, LC, EC run the experiments. MBi performed the bioinformatics analyses. MBa performed the MS experiments. CL and IS designed and generated the Lsh KO ESCs. MdD, LC, RD and FF wrote the manuscript. All the authors edited and approved the final manuscript.

\section{FUNDING}

MdD and LC were supported by the F.N.R.S. MBi was supported by the Télévie. This work was funded by grants from the Fonds de la Recherche Scientifique and Télévie, as well as by grants from the IUAP P7/03 program, the Action de Recherche Concerté (AUWB-2010-2015 ULB-No 7), the Belgian "Foundation against Cancer," the WB Health program, and the Fonds Gaston Ithier. Research in IS lab was supported by Cancer Research UK senior fellowship (C7215/A8983). 


\section{REFERENCES}

1. Robertson,K.D. (2001) DNA methylation, methyltransferases, and cancer. Oncogene, 20, 3139-3155.

2. Denis,H., Ndlovu,M.N. and Fuks,F. (2011) Regulation of mammalian DNA methyltransferases: a route to new mechanisms. EMBO Rep., 12, 647-56.

3. Ndlovu,M.N., Denis,H. and Fuks,F. (2011) Exposing the DNA methylome iceberg. Trends Biochem. Sci., 36, 381-7.

4. Tahiliani,M., Koh,K.P., Shen,Y., Pastor,W.A., Bandukwala,H., Brudno,Y., Agarwal,S., lyer,L.M., Liu,D.R., Aravind,L., et al. (2009) Conversion of 5-methylcytosine to 5hydroxymethylcytosine in mammalian DNA by MLL partner TET1. Science, 324, 9305 .

5. Ito,S., D'Alessio,A.C., Taranova,O.V., Hong,K., Sowers,L.C. and Zhang,Y. (2010) Role of Tet proteins in $5 \mathrm{mC}$ to $5 \mathrm{hmC}$ conversion, ES cell self-renewal, and ICM specification. Nature, 466, 1129-1133.

6. Kriaucionis,S. and Heintz,N. (2009) The nuclear DNA base, 5-hydroxymethylcytosine is present in brain and enriched in Purkinje neurons. Science, 324, 929-930.

7. Hahn,M.A., Szabó,P.E. and Pfeifer,G.P. (2014) 5-Hydroxymethylcytosine: A stable or transient DNA modification? Genomics, 104, 314-323.

8. Bachman,M., Uribe-Lewis,S., Yang,X., Williams,M., Murrell,A. and Balasubramanian,S. (2014) 5-Hydroxymethylcytosine is a predominantly stable DNA modification. Nat. Chem., 6, 1049-55.

9. Spruijt,C.G., Gnerlich,F., Smits,A.H., Pfaffeneder,T., Jansen,P.W.T.C., Bauer,C., Münzel,M., Wagner,M., Müller,M., Khan,F., et al. (2013) Dynamic readers for 5(hydroxy)methylcytosine and its oxidized derivatives. Cell, 152, 1146-59.

10. Deplus,R., Delatte,B., Schwinn,M.K.M.K., Defrance,M., Méndez,J., Murphy,N., Dawson,M.A.M.A., Volkmar,M., Putmans,P., Calonne,E., et al. (2013) TET2 and TET3 regulate GlcNAcylation and H3K4 methylation through OGT and SET1/COMPASS. EMBO J., 32, 645-55.

11. Delatte,B., Deplus,R. and Fuks,F. (2014) Playing TETris with DNA modifications. EMBO J., 33, 1198-211.

12. Blaschke,K., Ebata,K.T., Karimi,M.M., Zepeda-Martínez,J.A., Goyal,P., Mahapatra,S., Tam,A., Laird,D.J., Hirst,M., Rao,A., et al. (2013) Vitamin C induces Tet-dependent DNA demethylation and a blastocyst-like state in ES cells. Nature, 500, 222-226.

13. Chen,J., Guo,L., Zhang,L., Wu,H., Yang,J., Liu,H., Wang,X., Hu,X., Gu,T., Zhou,Z., et al. (2013) Vitamin $\mathrm{C}$ modulates TET1 function during somatic cell reprogramming. Nat. Genet., 45, 1504-1509.

14. Minor,E.A., Court,B.L., Young,J.I. and Wang,G. (2013) Ascorbate induces ten-eleven translocation (Tet) methylcytosine dioxygenase-mediated generation of 5hydroxymethylcytosine. J. Biol. Chem., 288, 13669-13674. 
15. Yin,R., Mao,S.-Q., Zhao,B., Chong,Z., Yang,Y., Zhao,C., Zhang,D., Huang,H., Gao,J., $\mathrm{Li}, \mathrm{Z}$., et al. (2013) Ascorbic acid enhances Tet-mediated 5-methylcytosine oxidation and promotes DNA demethylation in mammals. J. Am. Chem. Soc., 135, 1039610403.

16. Collignon,E., Canale,A., Al Wardi,C., Bizet,M., Calonne,E., Dedeurwaerder,S., Garaud,S., Naveaux,C., Barham,W., Wilson,A., et al. (2018) Immunity drives TET1 regulation in cancer through NF-kB. Sci. Adv., 4, eaap7309.

17. Cheng,J., Guo,S., Chen,S., Mastriano,S.J., Liu,C., D’Alessio,A.C., Hysolli,E., Guo,Y., Yao,H., Megyola,C.M., et al. (2013) An extensive network of TET2-targeting MicroRNAs regulates malignant hematopoiesis. Cell Rep., 5, 471-481.

18. Fu,X., Jin,L., Wang,X., Luo,A., Hu,J., Zheng,X., Tsark,W.M., Riggs,A.D., Ku,H.T. and Huang,W. (2013) MicroRNA-26a targets ten eleven translocation enzymes and is regulated during pancreatic cell differentiation. Proc. Natl. Acad. Sci. U. S. A., 110, 17892-17897.

19. Morita,S., Horii,T., Kimura,M., Ochiya,T., Tajima,S. and Hatada,I. (2013) miR-29 represses the activities of DNA methyltransferases and DNA demethylases. Int. J. Mol. Sci., 14, 14647-14658.

20. Song,S.J., Poliseno,L., Song,M.S., Ala,U., Webster,K., Ng,C., Beringer,G., Brikbak,N.J., Yuan,X., Cantley,L.C., et al. (2013) MicroRNA-antagonism regulates breast cancer stemness and metastasis via TET-family-dependent chromatin remodeling. Cell, 154, 311-24.

21. Hon,G.C., Hawkins,R.D., Caballero,O.L., Lo,C., Lister,R., Pelizzola,M., Valsesia,A., Ye,Z., Kuan,S., Edsall,L.E., et al. (2012) Global DNA hypomethylation coupled to repressive chromatin domain formation and gene silencing in breast cancer. Genome Res., 22, 246-258.

22. Ehrlich,M. (2009) DNA hypomethylation in cancer cells. Epigenomics, 1, 239-259.

23. Kosmider,O., Gelsi-Boyer,V., Slama,L., Dreyfus,F., Beyne-Rauzy,O., Quesnel,B., Hunault-Berger,M., Slama,B., Vey,N., Lacombe,C., et al. (2010) Mutations of IDH1 and IDH2 genes in early and accelerated phases of myelodysplastic syndromes and MDS/myeloproliferative neoplasms. Leukemia, 24, 1094-1096.

24. Figueroa,M.E., Abdel-Wahab,O., Lu,C., Ward,P.S., Patel,J., Shih,A., Li,Y., Bhagwat,N., Vasanthakumar,A., Fernandez,H.F., et al. (2010) Leukemic IDH1 and IDH2 Mutations Result in a Hypermethylation Phenotype, Disrupt TET2 Function, and Impair Hematopoietic Differentiation. Cancer Cell, 18, 553-567.

25. Lin,T.-L., Nagata,Y., Kao,H.-W., Sanada,M., Okuno,Y., Huang,C.-F., Liang,D.-C., Kuo,M.-C., Lai,C.-L., Lee,E.-H., et al. (2014) Clonal leukemic evolution in myelodysplastic syndromes with TET2 and IDH1/2 mutations. Haematologica, 99, 28-36.

26. Nettersheim,D., Heukamp,L.C., Fronhoffs,F., Grewe,M.J., Haas,N., Waha,A., Honecker,F., Waha,A., Kristiansen,G. and Schorle,H. (2013) Analysis of TET Expression/Activity and $5 \mathrm{mC}$ Oxidation during Normal and Malignant Germ Cell Development. PLOS ONE, 8, e82881. 
27. Geiman,T.M., Durum,S.K. and Muegge,K. (1998) Characterization of gene expression, genomic structure, and chromosomal localization of Hells (Lsh). Genomics, 54, 47783.

28. Geiman,T.M. and Muegge,K. (2000) Lsh, an SNF2/helicase family member, is required for proliferation of mature T lymphocytes. Proc. Natl. Acad. Sci. U. S. A., 97, 4772-7.

29. Clapier,C.R. and Cairns,B.R. (2009) The biology of chromatin remodeling complexes. Annu. Rev. Biochem., 78, 273-304.

30. Brzeski,J. and Jerzmanowski,A. (2003) Deficient in DNA methylation 1 (DDM1) defines a novel family of chromatin-remodeling factors. J. Biol. Chem., 278, 823-828.

31. Jenness,C., Giunta,S., Müller,M.M., Kimura,H., Muir,T.W. and Funabiki,H. (2018) HELLS and CDCA7 comprise a bipartite nucleosome remodeling complex defective in ICF syndrome. Proc. Natl. Acad. Sci. U. S. A., 115, E876-E885.

32. Geiman,T.M., Tessarollo,L., Anver,M.R., Kopp,J.B., Ward,J.M. and Muegge,K. Lsh, a SNF2 family member, is required for normal murine development. Biochim. Biophys. Acta BBA - Gen. Subj., 1526, 211-220.

33. Huang,J., Fan,T., Yan,Q., Zhu,H., Fox,S., Issaq,H.J., Best,L., Gangi,L., Munroe,D. and Muegge,K. (2004) Lsh, an epigenetic guardian of repetitive elements. Nucleic Acids Res., 32, 5019-28.

34. Dunican,D.S., Cruickshanks,H.A., Suzuki,M., Semple,C.A., Davey,T., Arceci,R.J., Greally,J., Adams,I.R. and Meehan,R.R. (2013) Lsh regulates LTR retrotransposon repression independently of Dnmt3b function. Genome Biol., 14, R146.

35. Tao,Y., Xi,S., Shan,J., Maunakea,A., Che,A., Briones,V., Lee,E.Y., Geiman,T., Huang,J., Stephens, R., et al. (2011) Lsh, chromatin remodeling family member, modulates genome-wide cytosine methylation patterns at nonrepeat sequences. Proc. Natl. Acad. Sci. U. S. A., 108, 5626-31.

36. Xi,S., Geiman,T.M., Briones,V., Tao,Y.G., Xu,H. and Muegge,K. (2009) Lsh Participates in DNA Methylation and Silencing of Stem Cell Genes. Stem Cells Dayt. Ohio, 27, 2691-2702.

37. Yu,W., Briones, V., Lister,R., McIntosh,C., Han,Y., Lee,E.Y., Ren,J., Terashima,M., Leighty,R.M., Ecker,J.R., et al. (2014) CG hypomethylation in Lsh-/- mouse embryonic fibroblasts is associated with de novo H3K4me1 formation and altered cellular plasticity. Proc. Natl. Acad. Sci. U. S. A., 111, 5890-5895.

38. Yan,Q., Huang,J., Fan,T., Zhu,H. and Muegge,K. (2003) Lsh, a modulator of CpG methylation, is crucial for normal histone methylation. EMBO J., 22, 5154-62.

39. Zhu,H., Geiman,T.M., Xi,S., Jiang,Q., Schmidtmann,A., Chen,T., Li,E. and Muegge,K. (2006) Lsh is involved in de novo methylation of DNA. EMBO J., 25, 335-45.

40. Myant,K. and Stancheva,I. (2008) LSH cooperates with DNA methyltransferases to repress transcription. Mol. Cell. Biol., 28, 215-26.

41. Ren,J., Briones, V., Barbour,S., Yu,W., Han,Y., Terashima,M. and Muegge,K. (2015) The ATP binding site of the chromatin remodeling homolog Lsh is required for nucleosome density and de novo DNA methylation at repeat sequences. Nucleic Acids Res., 43, 1444-55. 
42. Suzuki,T., Farrar,J.E., Yegnasubramanian,S., Zahed,M., Suzuki,N. and Arceci,R.J. (2008) Stable knockdown of PASG enhances DNA demethylation but does not accelerate cellular senescence in TIG-7 human fibroblasts. Epigenetics, 3, 281-91.

43. Myant,K., Termanis,A., Sundaram,A.Y.M., Boe,T., Li,C., Merusi,C., Burrage,J., de Las Heras,J.I. and Stancheva,I. (2011) LSH and G9a/GLP complex are required for developmentally programmed DNA methylation. Genome Res., 21, 83-94.

44. Ren,J., Finney,R., Ni,K., Cam,M. and Muegge,K. (2019) The chromatin remodeling protein Lsh alters nucleosome occupancy at putative enhancers and modulates binding of lineage specific transcription factors. Epigenetics, 14, 277-293.

45. Schnütgen,F., De-Zolt,S., Van Sloun,P., Hollatz,M., Floss,T., Hansen,J., Altschmied,J., Seisenberger,C., Ghyselinck,N.B., Ruiz,P., et al. (2005) Genomewide production of multipurpose alleles for the functional analysis of the mouse genome. Proc. Natl. Acad. Sci. U. S. A., 102, 7221-7226.

46. Viré,E., Brenner,C., Deplus,R., Blanchon,L., Fraga,M., Didelot,C., Morey,L., Van Eynde,A., Bernard,D., Vanderwinden,J.-M., et al. (2006) The Polycomb group protein EZH2 directly controls DNA methylation. Nature, 439, 871-4.

47. Berlivet,S., Guiraud,V., Houlard,M. and Gérard,M. (2007) pHYPER, a shRNA vector for high-efficiency RNA interference in embryonic stem cells. BioTechniques, 42, 738, 740-743.

48. von Eyss,B., Maaskola,J., Memczak,S., Möllmann,K., Schuetz,A., Loddenkemper,C., Tanh,M.-D., Otto,A., Muegge,K., Heinemann,U., et al. (2012) The SNF2-like helicase HELLS mediates E2F3-dependent transcription and cellular transformation. EMBO J., 31, 972-85.

49. Fuks,F., Burgers,W.A., Brehm,A., Hughes-Davies,L. and Kouzarides, T. (2000) DNA methyltransferase Dnmt1 associates with histone deacetylase activity. Nat. Genet., 24, 88-91.

50. Ye,T., Krebs,A.R., Choukrallah,M.-A., Keime,C., Plewniak,F., Davidson,I. and Tora,L. (2011) seqMINER: an integrated ChIP-seq data interpretation platform. Nucleic Acids Res., 39, e35-e35.

51. High Protein Yield and Purity with the HaloTag® Protein Purification System. (2010) Httpfrancepromegacomresourcespubhubhigh-Protein-Yield--Purity---Halotag-ProteinPurif.-Syst.

52. Ciccarone,F., Valentini,E., Zampieri,M. and Caiafa,P. (2015) 5mC-hydroxylase activity is influenced by the PARylation of TET1 enzyme. Oncotarget, 6, 24333-24347.

53. Zheng,L., Zhai,Y., Li,N., Ma,F., Zhu,H., Du,X., Li,G. and Hua,J. (2016) The Modification of Tet1 in Male Germline Stem Cells and Interact with PCNA, HDAC1 to promote their Self-renewal and Proliferation. Sci. Rep., 6, 37414.

54. Niu,X., Zhang,T., Liao,L., Zhou,L., Lindner,D.J., Zhou,M., Rini,B., Yan,Q. and Yang,H. (2012) The von Hippel-Lindau tumor suppressor protein regulates gene expression and tumor growth through histone demethylase JARID1C. Oncogene, 31, 776-86.

55. Muegge,K. (2005) Lsh, a guardian of heterochromatin at repeat elements. Biochem. Cell Biol. Biochim. Biol. Cell., 83, 548-54. 
56. Dunican,D.S., Pennings,S. and Meehan,R.R. (2015) Lsh Is Essential for Maintaining Global DNA Methylation Levels in Amphibia and Fish and Interacts Directly with Dnmt1. BioMed Res. Int., 10.1155/2015/740637.

57. Han,Y., Ren,J., Lee,E., Xu,X., Yu,W. and Muegge,K. (2017) Lsh/HELLS regulates selfrenewal/proliferation of neural stem/progenitor cells. Sci. Rep., 7, 1136.

58. Sun,L.-Q., Lee,D.W., Zhang,Q., Xiao,W., Raabe,E.H., Meeker,A., Miao,D., Huso,D.L. and Arceci,R.J. (2004) Growth retardation and premature aging phenotypes in mice with disruption of the SNF2-like gene, PASG. Genes Dev., 18, 1035-46.

59. Fan,T., Schmidtmann,A., Xi,S., Briones,V., Zhu,H., Suh,H.C., Gooya,J., Keller,J.R., $\mathrm{Xu}, \mathrm{H}$., Roayaei,J., et al. DNA hypomethylation caused by Lsh deletion promotes erythroleukemia development. Epigenetics, 3, 134-42.

60. Dennis,K., Fan,T., Geiman,T., Yan,Q. and Muegge,K. (2001) Lsh, a member of the SNF2 family, is required for genome-wide methylation. Genes Dev., 15, 2940-2944.

61. Chronis,C., Fiziev,P., Papp,B., Butz,S., Bonora,G., Sabri,S., Ernst,J. and Plath,K. (2017) Cooperative Binding of Transcription Factors Orchestrates Reprogramming. Cell, 168, 442-459.e20.

62. Dunican,D.S., Cruickshanks,H.A., Suzuki,M., Semple,C.A., Davey,T., Arceci,R.J., Greally,J., Adams,I.R. and Meehan,R.R. (2013) Lsh regulates LTR retrotransposon repression independently of Dnmt3b function. Genome Biol., 14, R146.

63. Sun,W., Zang,L., Shu,Q. and Li,X. (2014) From development to diseases: the role of 5hmC in brain. Genomics, 104, 347-51.

64. Kraus,T.F.J., Guibourt,V. and Kretzschmar,H.A. (2015) 5-Hydroxymethylcytosine, the 'Sixth Base', during brain development and ageing. J. Neural Transm. Vienna Austria 1996, 122, 1035-1043.

65. Amouroux,R., Nashun,B., Shirane,K., Nakagawa,S., Hill,P.W.S., D’Souza,Z., Nakayama,M., Matsuda,M., Turp,A., Ndjetehe,E., et al. (2016) De novo DNA methylation drives $5 \mathrm{hmC}$ accumulation in mouse zygotes. Nat. Cell Biol., $10.1038 /$ ncb3296.

66. Tan,L. and Shi,Y.G. (2012) Tet family proteins and 5-hydroxymethylcytosine in development and disease. Dev. Camb. Engl., 139, 1895-1902.

67. Delatte,B. and Fuks,F. (2013) TET proteins: on the frenetic hunt for new cytosine modifications. Brief. Funct. Genomics, 12, 191-204.

68. Nestor,C.E., Reddington,J.P., Benson,M. and Meehan,R.R. (2014) Investigating 5hydroxymethylcytosine $(5 \mathrm{hmC})$ : the state of the art. Methods Mol. Biol. Clifton NJ, 1094, 243-258.

69. Jia,J., Shi,Y., Chen,L., Lai,W., Yan,B., Jiang,Y., Xiao,D., Xi,S., Cao,Y., Liu,S., et al. (2017) Decrease in Lymphoid Specific Helicase and 5-hydroxymethylcytosine Is Associated with Metastasis and Genome Instability. Theranostics, 7, 3920-3932.

70. Jia,J., Shi,Y., Chen,L., Lai,W., Yan,B., Jiang,Y., Xiao,D., Xi,S., Cao,Y., Liu,S., et al. (2017) Decrease in Lymphoid Specific Helicase and 5-hydroxymethylcytosine Is Associated with Metastasis and Genome Instability. Theranostics, 7. 
71. Jia,G., Fu,Y., Zhao,X., Dai,Q., Zheng,G., Yang,Y.Y.-G., Yi,C., Lindahl,T., Pan,T., Yang,Y.Y.-G., et al. (2011) N6-methyladenosine in nuclear RNA is a major substrate of the obesity-associated FTO. Nat. Chem. Biol., 7, 885-7.

72. Lee,D.W., Zhang,K., Ning,Z.Q., Raabe,E.H., Tintner,S., Wieland,R., Wilkins,B.J., Kim,J.M., Blough,R.I. and Arceci,R.J. (2000) Proliferation-associated SNF2-like gene (PASG): a SNF2 family member altered in leukemia. Cancer Res., 60, 3612-22.

73. Moran-Crusio,K., Reavie,L., Shih,A., Abdel-Wahab,O., Ndiaye-Lobry,D., Lobry,C., Figueroa,M.E., Vasanthakumar,A., Patel,J., Zhao,X., et al. (2011) Tet2 loss leads to increased hematopoietic stem cell self-renewal and myeloid transformation. Cancer Cell, 20, 11-24.

74. Quivoron,C., Couronné,L., Della Valle,V., Lopez,C.K., Plo,I., Wagner-Ballon,O., Do Cruzeiro,M., Delhommeau,F., Arnulf,B., Stern,M.-H., et al. (2011) TET2 inactivation results in pleiotropic hematopoietic abnormalities in mouse and is a recurrent event during human lymphomagenesis. Cancer Cell, 20, 25-38.

75. Williams,K., Christensen,J., Pedersen,M.T., Johansen,J. V, Cloos,P.A.C., Rappsilber,J. and Helin,K. (2011) TET1 and hydroxymethylcytosine in transcription and DNA methylation fidelity. Nature, 473, 343-8.

76. Wu,H., D'Alessio,A.C., Ito,S., Xia,K., Wang,Z., Cui,K., Zhao,K., Sun,Y.E. and Zhang,Y. (2011) Dual functions of Tet1 in transcriptional regulation in mouse embryonic stem cells. Nature, 473, 389-93.

77. Xue,S., Liu,C., Sun,X., Li,W., Zhang,C., Zhou,X., Lu,Y., Xiao,J., Li,C., Xu,X., et al. (2016) TET3 Inhibits Type I IFN Production Independent of DNA Demethylation. Cell Rep., 16, 1096-1105. 


\section{FIGURE LEGENDS}

\section{Figure 1: LSH is a TET interacting partner.}

(A) Halo pulldowns were performed from HEK293T cells overexpressing indicated Halo-tagged Tet protein. Spectral counts for LSH, SIN3A and OGT interacting proteins are indicated for biological replicates. As previously reported, TET1, TET3 but not TET2, shows interaction with SIN3A (75-77) and OGT interacts with all TETs, most abundantly with TET2 and TET3 (10). LSH interacts with TET1 and TET3. EEF1D (Isoform 1 of Elongation factor 1-delta) is a negative control.

(B) Following transfection of the indicated FLAG-tagged constructs in HEK293T, cell extracts were precipitated with the anti-FLAG antibody and the presence of endogenous LSH was detected with anti-LSH antibody (right panel). Endogenous LSH was used as an input loading control and overexpression of TET catalytic domains (CD) is shown (left panel).

(C) Endogenous co-IPs in ES cells. Cell lysates were subjected to immunoprecipitation with an anti-Tet1 (left panel) or anti-Tet2 (right panel) antibody and subjected to western blot using anti-Lsh or the indicated antibodies as control. Inputs and IP controls are shown. Vertical lines indicate juxtaposition of lanes within the same blot, exposed for the same time.

\section{Figure 2: Lsh knock-out decreases 5hmC global level in ES and MEF cells.}

(A) Dot blot quantification of 5hmC global level in genomic DNA from wild-type and Lsh KO ES cells. ssDNA was used as loading control. Results were quantified using ImageJ. Error bars indicate s.d. of three biological replicates with a representative blot shown.

(B) MS quantifications of $5 \mathrm{hmC}$ (left panel) and $5 \mathrm{mC}$ (right panel) in wild-type and Lsh KO ES. Error bars indicate s.d. of three biological replicates.

(C) Dot blot quantification of 5hmC global level in genomic DNA from wild-type and Lsh KO MEF cells. ssDNA was used as loading control. Results were quantified using ImageJ. Error bars indicate s.d. of three biological replicates with a representative blot shown. 
(D) MS quantifications of $5 \mathrm{hmC}$ (left panel) and $5 \mathrm{mC}$ (right panel) in wild-type and Lsh KO MEFs. Error bars indicate s.d. of three biological replicates.

\section{Figure 3: 5hmC level is impaired in Lsh KO ES cells at specific genomic loci.}

(A) Pie chart showing the percentage of up and down $5 \mathrm{hmC}$ regions in ES cells upon Lsh loss (8557 regions in total).

(B) Distribution of differentially hydroxymethylated regions on genomic features: promoters, gene body, enhancer, multiple or mixed features and intergenic. In color code are observed number whereas in grey are the expected numbers.

(C) Representative UCSC Genome Browser plot from hMeDIP-seq data in ES cells (Podxl2 gene).

(D) An analyzed subset of differentially hydroxymethylated regions. qPCR analysis of hyper-hydroxymethylated genes (Sc/36, GM5122, Zfat and Alox15 genes) and on hypo-hydroxymethylated genes (Mf151, Hspa, Mef2d and Gbj5) after hMeDIP. IP/input represents real-time qPCR values normalized with respect to the input chromatin \pm relative error of 3 independent experiments. Regions with no changes in $5 \mathrm{hmC}$ level are shown as negative controls (Ctrl1 \& Ctrl2).

Figure 4: $5 \mathrm{hmC}$ level is impaired in Lsh KO MEFs cells at specific genomic loci.

(A) Pie chart showing the percentage of up and down $5 \mathrm{hmC}$ in MEF cells upon Lsh loss (9002 regions in total).

(B) Distribution of differentially hydroxymethylated regions on genomic features: promoters (113), gene body (4504), enhancer (26), multiple or mixed features (652) and intergenic (3707). In color code are observed number whereas in grey are the expected numbers.

(C) Representative UCSC Genome Browser plot from hMeDIP-seq data in MEF cells (Btg4 gene).

(D) An analyzed subset of differentially hydroxymethylated regions. qPCR analysis of hyper-hydroxymethylated genes (Bdnf, Btg4, Elfn2 and Fam92b genes) and on hypohydroxymethylated genes (Hp, Gdf5, Klf2 and Sfi1 genes) after hMeDIP. IP/input 
represents real-time qPCR values normalized with respect to the input chromatin \pm relative error of 3 independent experiments. Regions with no changes in $5 \mathrm{hmC}$ level are shown as negative controls (Ctrl1 \& Ctrl2).

Figure 5: 5hmC differences are not a direct consequence of $5 \mathrm{mC}$ modifications upon Lsh loss and are not always followed by direct gene expression change

(A) Pie chart representing the percentage of regions displaying changes in $5 \mathrm{hmC}$ level only, in $5 \mathrm{mC}$ level only or in $5 \mathrm{hmC}$ and $5 \mathrm{mC}$ level. Comparison was made between our $5 \mathrm{hmC}$ sequencing data and public available data from MeDIP followed by genome-wide tiling arrays in wild-type versus Lsh KO MEFs (43).

(B) MeDIP qPCR experiments on target genes showing $5 \mathrm{hmC}$ increase, decrease or no $5 \mathrm{hmC}$ (control regions) in our high throughput sequencing data. Results are presented as percentages of Input \pm relative error of 3 independent experiments.

(C) Venn diagram showing overlap between the differentially hydroxymethylated genes identified by hMeDIP-seq (this study, see supp table 1) and differentially expressed genes (43). P-value overlap is 0.080300 showing no statistical relevance. Lower panel shows the mRNA up or down regulation of the 479 common genes.

(D) Heatmap showing 7 histone modifications (H3K4me3, H3K4me2, H3K9Ac, H3K27Ac, H3K27me3, H3K79me2, H3K36me3) and one histone variant (H3.3) occupancy at the 9002 DhMRs defining 6 different sub-groups. 


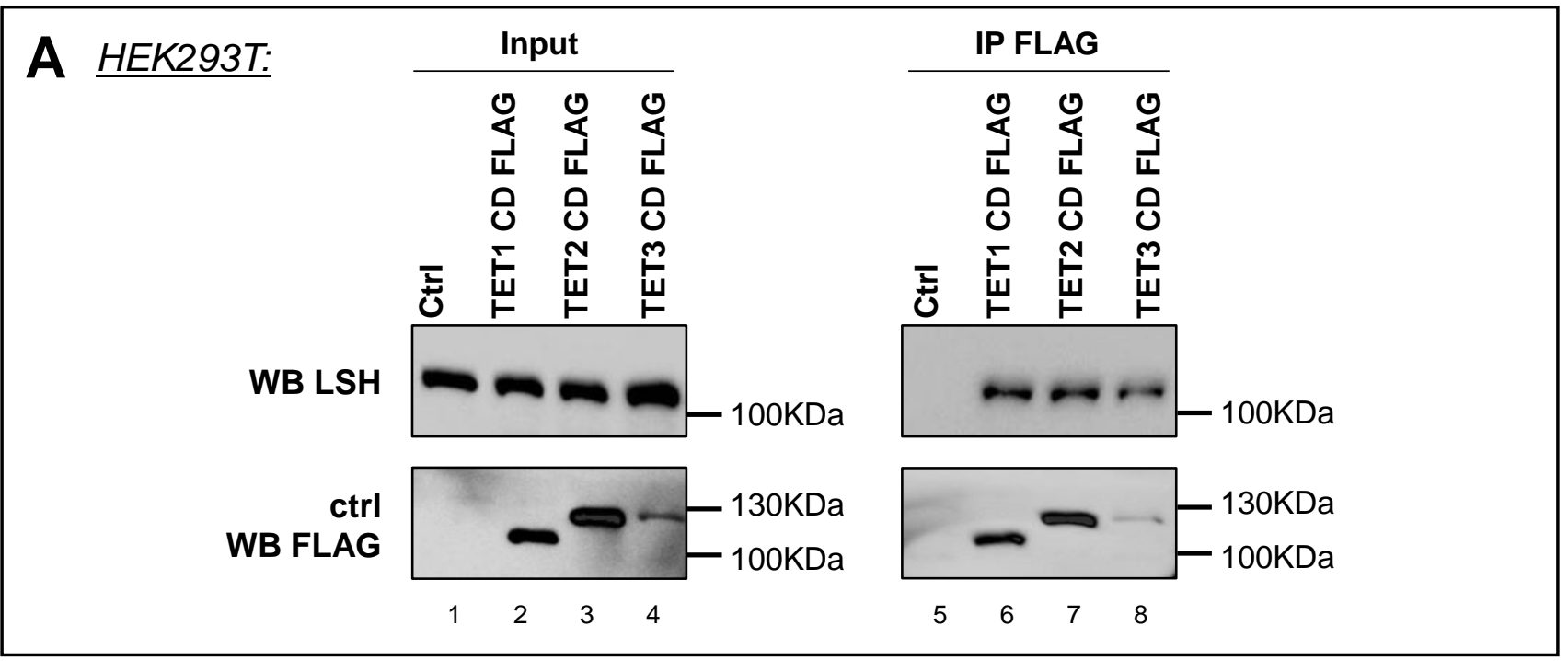

B ESC:

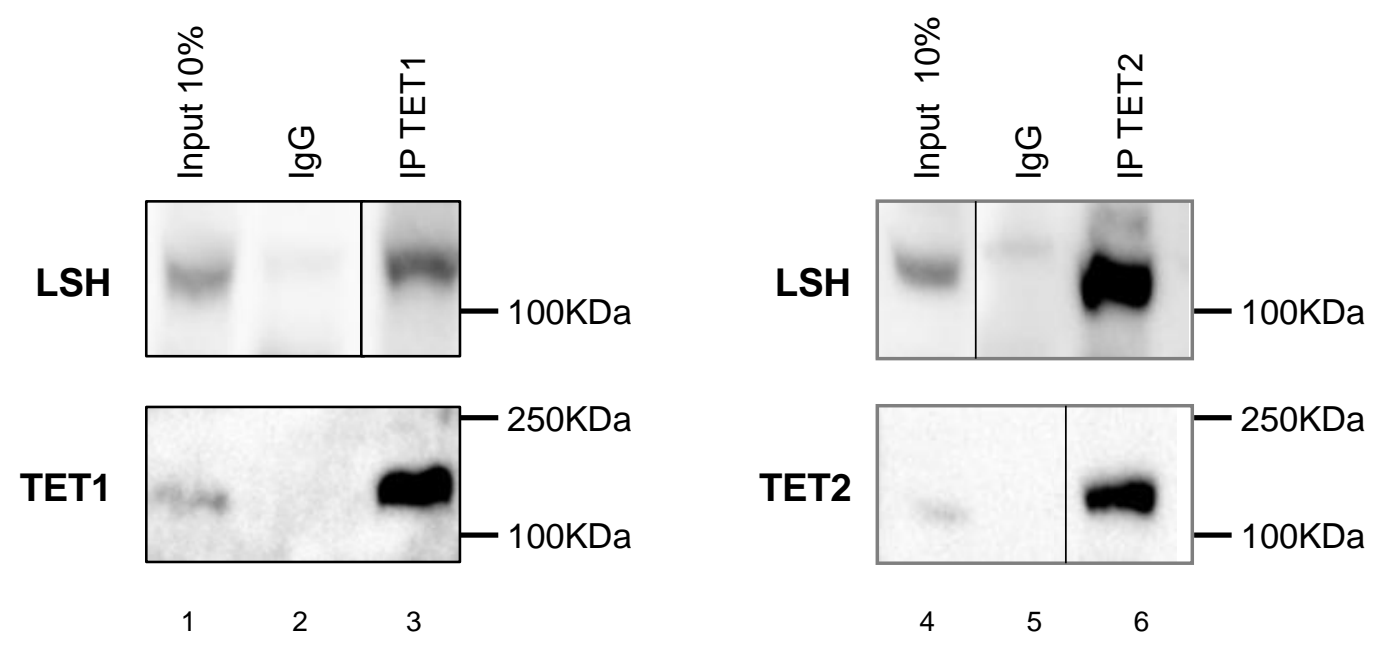


A
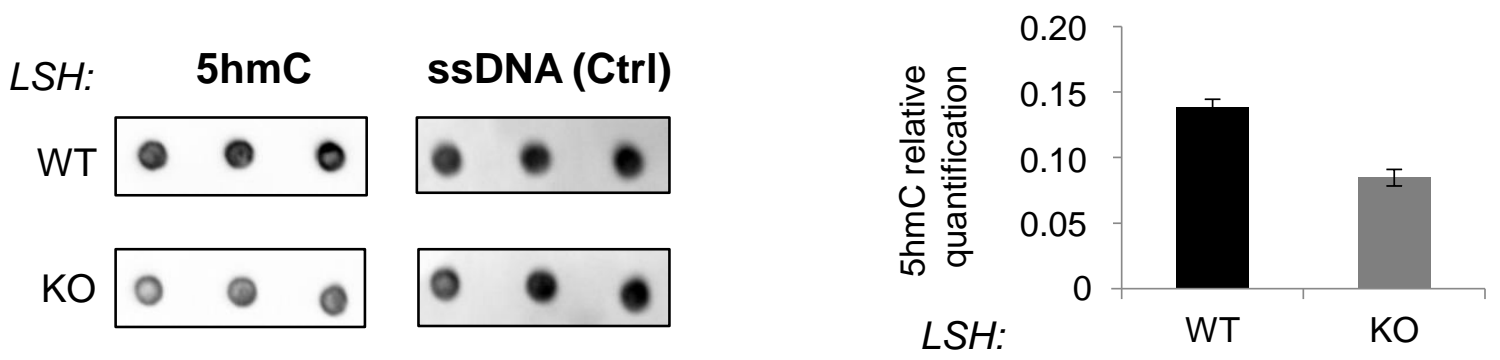

B

$5 \mathrm{hmC}$

\section{Mass spectrometry (ES)}

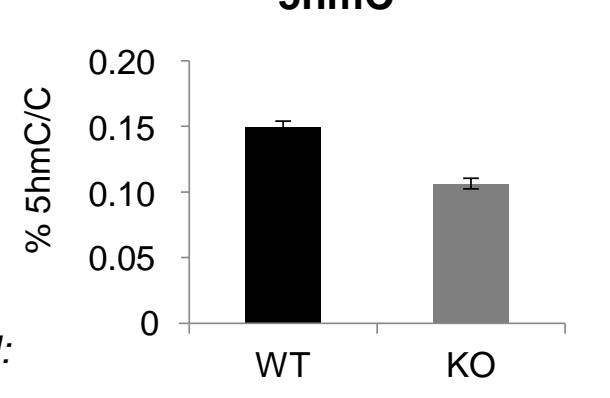

\section{$\underline{\text { Dot Blot (MEF) }}$}

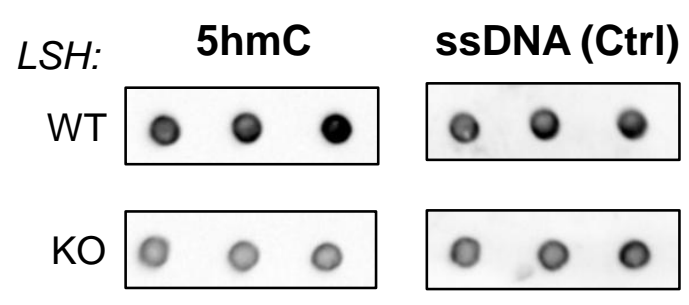

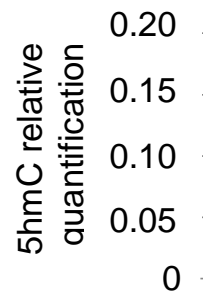

$L S H:$
$5 \mathrm{mC}$

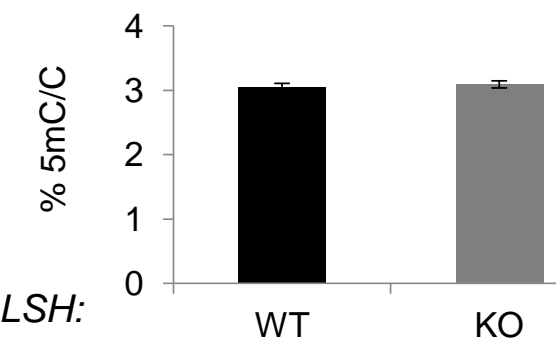

D

$L S H$ :

\section{Mass spectrometry (MEF)}

$5 \mathrm{hmC}$

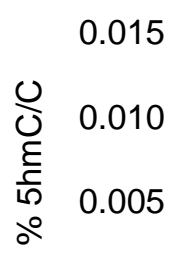

0

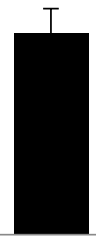

WT

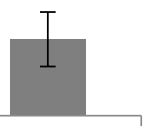

$\mathrm{KO}$

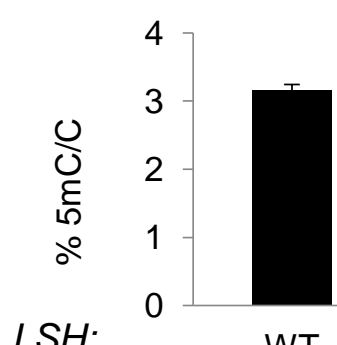

WT
$5 \mathrm{mC}$

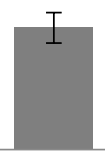

$\mathrm{KO}$ 
hMeDIP-seq in ESC

A

Differential $5 \mathrm{hmC}$ regions

8557 regions

ESC:

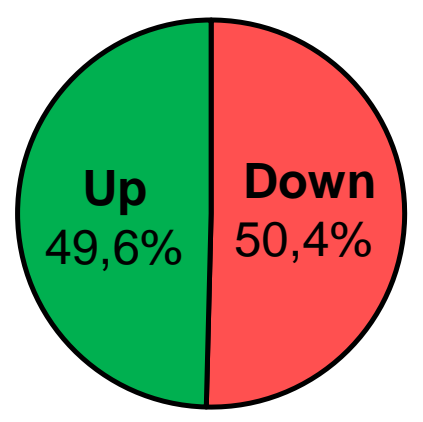

B

Observed

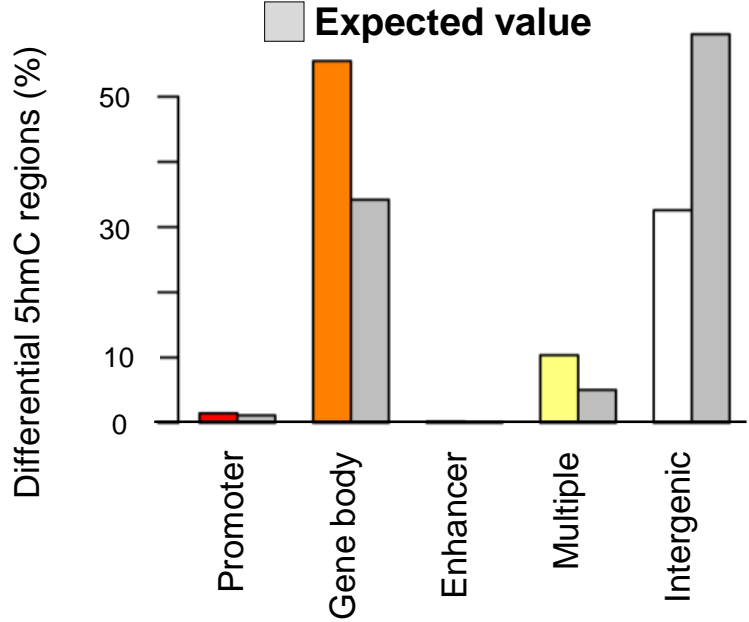

\section{C}

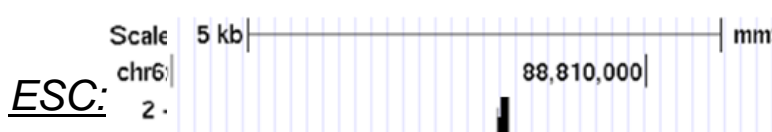

WT
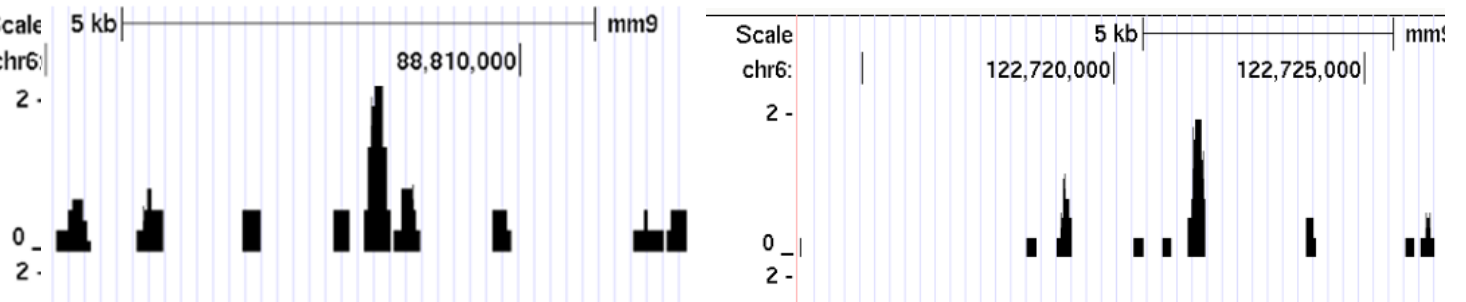

LSH KO

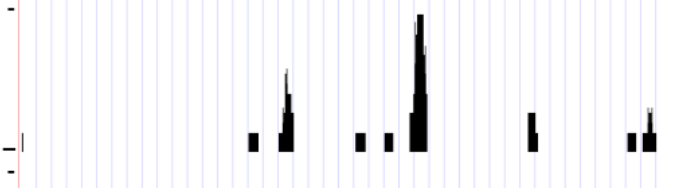

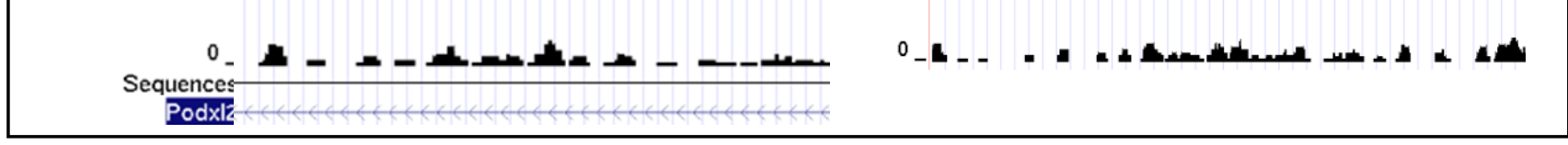

D

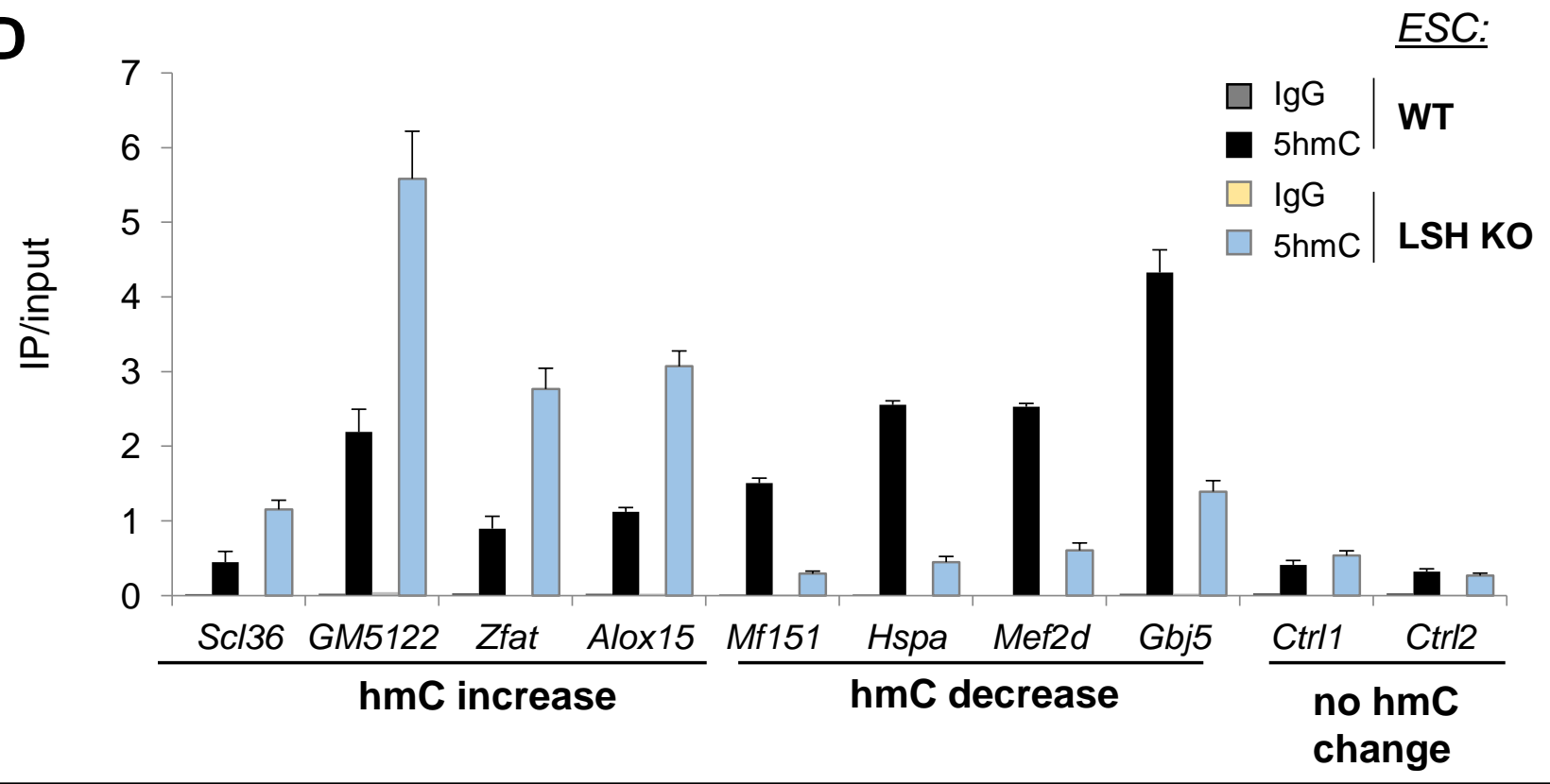


hMeDIP-seq in MEF

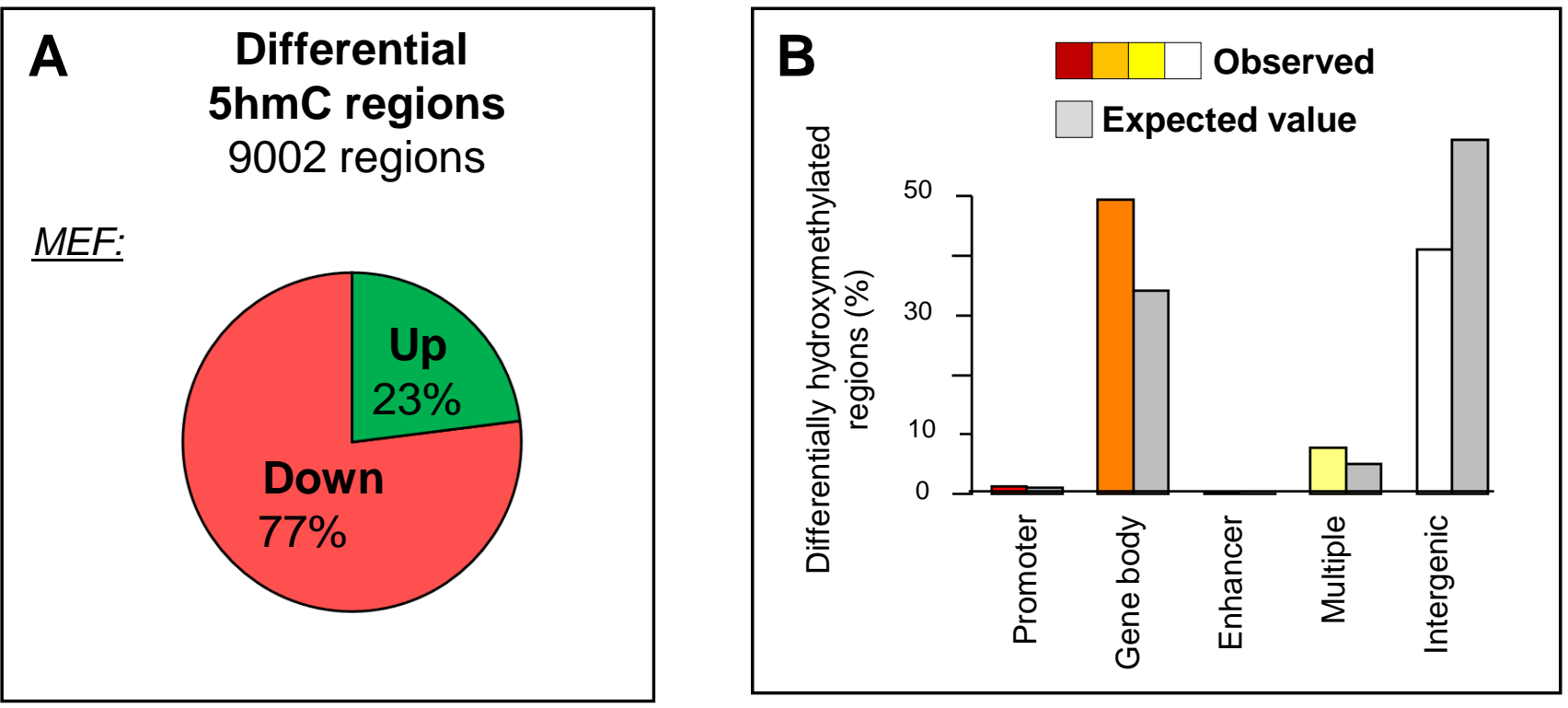
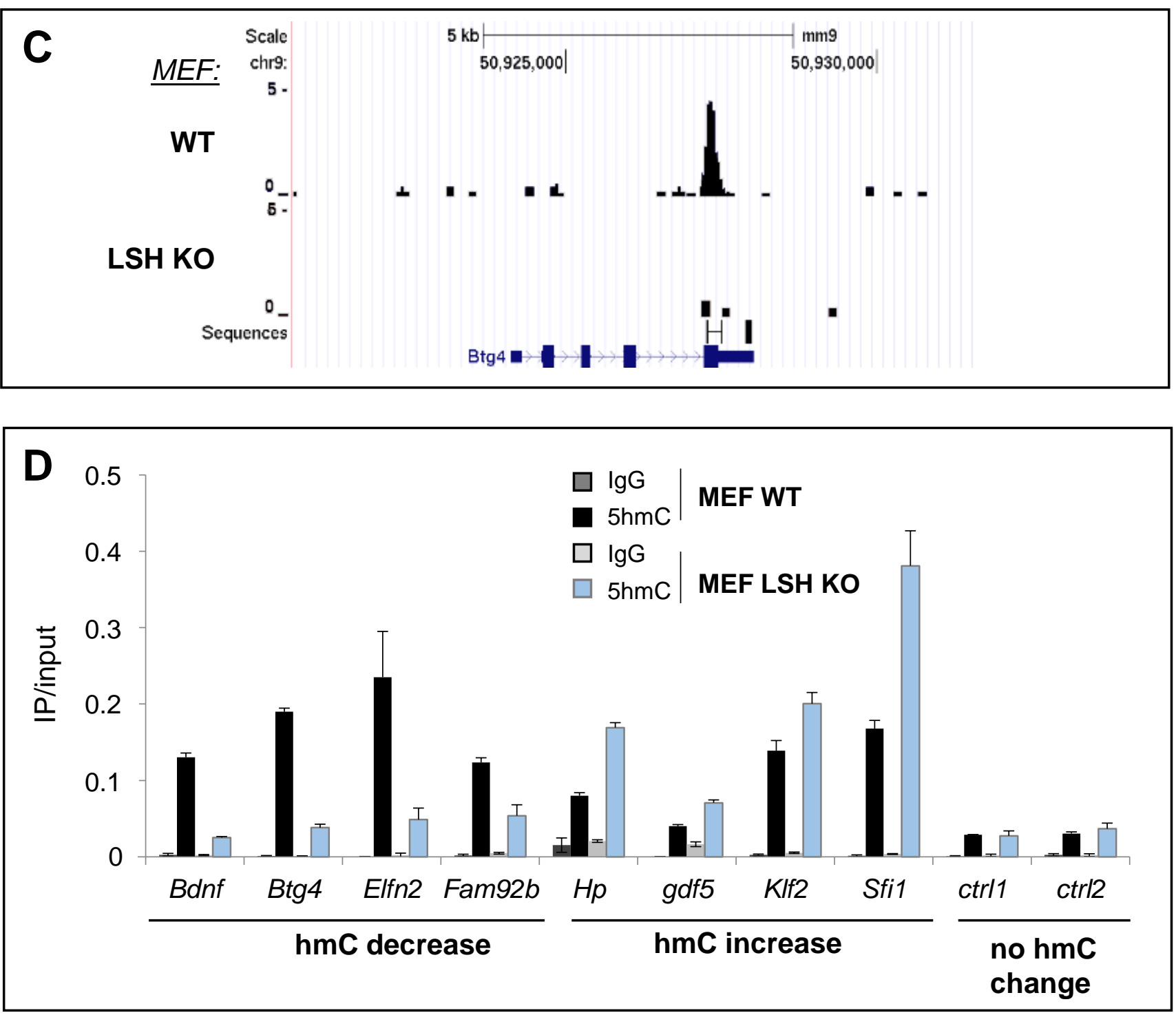
$\underline{\text { Hydroxymethylome and methylome comparision }}$
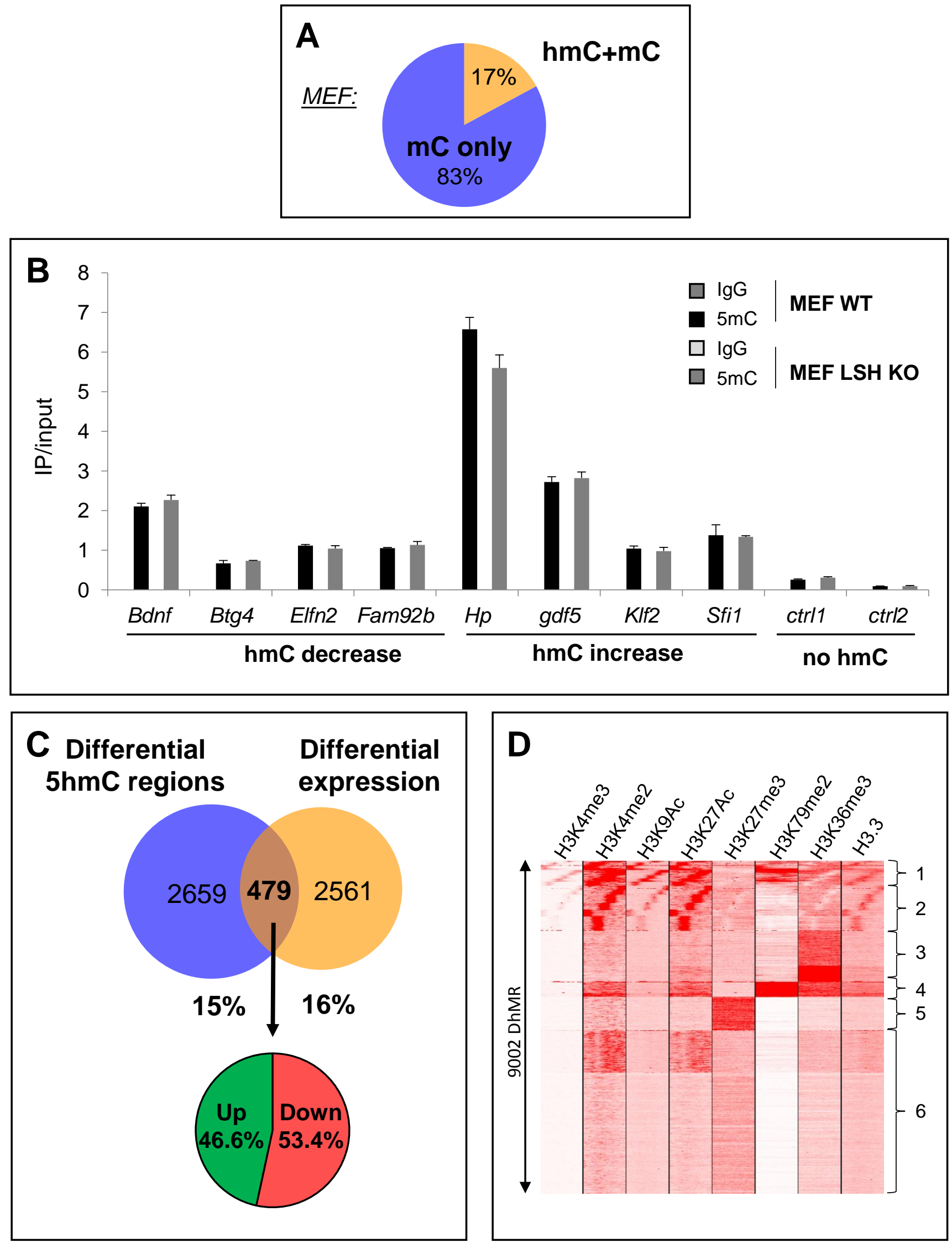